\title{
TALK Score: Development and Validation of a Prognostic Model for Predicting Larynx Preservation Outcome
}

\author{
Eric J. Sherman, MD; Susan G. Fisher, PhD; Dennis H. Kraus, MD; Michael J. Zelefsky, MD; \\ Venkatraman E. Seshan, PhD; Bhuvanesh Singh, MD; Ashok R. Shaha, MD; Jatin P. Shah, MD; \\ Gregory T. Wolf, MD; David G. Pfister, MD
}

\begin{abstract}
Objectives/Hypothesis: To develop and validate a simple prognostic tool that would help predict larynx preservation outcome.

Study Design: A retrospective review of 3 prospective studies.

Methods: We reviewed consecutive chemotherapy/radiation protocols for patients $(\mathrm{n}=170)$ with advanced, resectable, squamous cell, larynx, or pharynx cancer treated at Memorial Sloan-Kettering Cancer Center from 1988 to 1995 with larynx preservation intent. The outcome was successful larynx preservation. Model validation used data from U. S. Department of Veterans Affairs larynx preservation study.

Results: The developed model added one point for each poor prognostic covariate present (show in parentheses) and was given the acronym TALK: T stage (T4), albumin $(<4 \mathrm{~g} / \mathrm{dL})$, maximum alcohol/liquor use $(\geq 6$ drinks/day or heavy drinking), and Karnofsky performance status $(<80 \%)$. The 3 -year larynx preservation rates by TALK score were $65 \%(0), 41 \%$ (1-2), and 6\% (3-4), $P<.0001$; on validation, the TALK 3-4 group was particularly well demarcated.
\end{abstract}

Conclusions: The TALK score is an easily applied and valid tool that should assist treatment selection.

Key Words: Laryngeal neoplasms, decision making, prognosis, speech, combined modality therapy.

Level of Evidence: $2 c$.

Laryngoscope, 122:1043-1050, 2012

\section{INTRODUCTION}

Most advanced cancers of the larynx and surrounding structures require total laryngectomy if managed with primary surgery. This operation is among those most feared by patients. ${ }^{1}$ Both voice alteration and related communication challenges, as well as the presence of a stoma may adversely affect quality of life. ${ }^{2,3}$ Voice rehabilitation strategies exist, but many patients feel totally or partly dissatisfied with the options and continue to feel restricted in their daily lives. ${ }^{4}$ Accordingly, the development of treatment programs intended to preserve laryngeal function without compromising

From the Division of Solid Tumor Oncology, Department of Medicine (E.J.S., D.G.P.), the Head and Neck Service, Department of Surgery (D.H.K., B.S., A.R.S., J.P.S.), the Brachytherapy Service, Department of Radiation Oncology (M.J.z.), and the Department of Biostatistics (v.E.s.), Memorial Sloan-Kettering Cancer Center, New York, New York; the Department of Otolaryngology (G.T.w.), University of Michigan Health System, Ann Arbor, Michigan; and the University of Rochester Medical Center (s.G.F.), Rochester, New York, U.S.A.

Editor's Note: This Manuscript was accepted for publication January 3,2012 .

Presented in part at the 39th Annual Meeting of the American Society of Clinical Oncology, Chicago, Illinois, U.S.A., May 31 - June 3, 2003.

Supported in part by the Overman and Garban Intercapital Funds. The authors have no other funding, financial relationships, or conflicts of interest to disclose.

Send correspondence to Eric J. Sherman, MD, Memorial SloanKettering Cancer Center, Division of Solid Tumor Oncology, Box 188, 1275 York Avenue, New York, NY 10065. E-mail: shermane@mskcc.org

DOI: 10.1002/lary.23220 survival is an important priority within head and neck oncology and of great interest to patients.

Historically, conservation surgery ${ }^{5,6}$ and radiation ${ }^{7}$ as a single modality had been the principal larynx preservation options. In the last 15 years, integrated chemotherapy/radiotherapy has established itself as a broadly applicable larynx preservation strategy. Randomized trials have shown that such an approach offers voice preservation and improved quality of life without compromise in survival. ${ }^{8-10}$

When successful organ preservation is achieved, chemotherapy/radiation is generally viewed as having been the appropriate treatment choice for the patient; unfortunately, in some patients the strategy will fail. In this setting, a patient would have experienced the potential toxicities of chemotherapy (e.g., neuropathy, hearing loss) and definitive-dose radiation therapy (e.g., increased risk of severe mucositis, feeding tube) and the potential added morbidity associated with surgery after those treatments without any apparent benefit and many added costs. Arguably, such patients would have been better served by proceeding directly to primary total laryngectomy. Understandably, knowing whether a given patient has a higher or lower likelihood of success with chemotherapy/radiation therapy would be useful to both physicians and patients.

There is currently no validated methodology available to help separate better from poorer risk patients before the initiation of larynx preservation therapy. Therefore, treatment choice is often subjective and may use widely varying, difficult-to-reproduce criteria. Our 
TABLE I.

Induction Chemotherapy Regimens.

\begin{tabular}{|c|c|c|}
\hline Drugs & Dosage & Days \\
\hline \multicolumn{3}{|c|}{ Protocol 1988-1990 $(n=70)$} \\
\hline Cisplatin & $100 \mathrm{mg} / \mathrm{m}^{2} \mathrm{IV}$ & $1,29,57$ \\
\hline 5-fluorouracil & $1,000 \mathrm{mg} / \mathrm{m}^{2} \mathrm{CIV}$ & / 1-5, 29-33, 57-61 \\
\hline \multicolumn{3}{|c|}{ Protocol 1991-1995 $(n=103)$} \\
\hline Cisplatin $(n=22)$ & $100 \mathrm{mg} / \mathrm{m}^{2} \mathrm{IV}$ & $1,22,43$ \\
\hline $\begin{array}{l}\text { 5-fluorouracil } \\
\quad(\mathrm{n}=22)\end{array}$ & $800 \mathrm{mg} / \mathrm{m}^{2} \mathrm{CIV}$ & $1-5,22-26,43-47$ \\
\hline $\begin{array}{l}\text { Leucovorin calcium } \\
\quad(n=22)\end{array}$ & $\begin{array}{l}100 \mathrm{mg} P O \\
\text { every } 4 \mathrm{hr}\end{array}$ & $1-5,22-26,43-47$ \\
\hline Cisplatin $(n=81)$ & $100 \mathrm{mg} / \mathrm{m}^{2} \mathrm{IV}$ & $1,22,43$ \\
\hline $\begin{array}{l}\text { 5-fluorouracil } \\
\quad(\mathrm{n}=81)\end{array}$ & \multicolumn{2}{|c|}{$1,000 \mathrm{mg} / \mathrm{m}^{2}$ CIV $1-5,22-26,43-47$} \\
\hline
\end{tabular}

$\mathrm{IV}=$ intravenous; $\mathrm{CIV}=$ continuous intravenous; $\mathrm{PO}=$ orally.

objective was to create a simple prognostic tool using readily available clinical data that would help predict successful and, perhaps more importantly, unsuccessful larynx preservation outcome to facilitate treatment decision making in this setting.

\section{MATERIALS AND METHODS}

\section{Patients}

From 1988 to 1995, 173 patients with histologically confirmed squamous cell carcinoma of the larynx, hypopharynx, or oropharynx were treated on one of two consecutive, institutional review board-approved protocols at Memorial Sloan-Kettering Cancer Center with the primary intent of preserving the larynx. Seventy patients participated in the first protocol (1988-1990), and 103 patients participated in the second protocol (1991-1995). Three patients were found to be ineligible ( 2 with nonsquamous cell histology; 1 with $\mathrm{M}_{1}$ disease before initial treatment) and were excluded. Therefore, a total of 170 patients were included in this study. All histologic material was reviewed by the department of pathology at Memorial Hospital. Entry criteria were similar among the studies and have been previously described. ${ }^{11-13}$ All patients gave informed consent after the studies were approved by the local human investigations committee.

\section{Treatment}

The details of treatment have been previously discussed ${ }^{11-13}$ and are summarized in Table I and II. In both studies, three cycles of induction cisplatin-based combination chemotherapy were planned with appropriate supportive measures. ${ }^{14}$ The decision whether to proceed to radiation-based treatment or primary surgical management depended on the extent of response to chemotherapy. In general, patients with a major response at the primary site and no progression in the neck proceeded to radiation-based treatment; for lesser response categories and progression, surgical intervention was recommended. The patients on the first protocol received conventional external-beam radiation that was delivered in 1.8- to 2-Gy fractions to a planned total dose of 66 to 70 Gy to the primary site and other sites of gross disease. The patients on the second protocol received delayed accelerated external-beam radiation therapy that was delivered in 1.8-Gy daily fractions during weeks 1 to 4 , followed by twice-daily fractions (1.8 Gy in the morning and 1.6 Gy in the afternoon) during weeks 5 to 6 , for a total planned dose of $70 \mathrm{~Gy}$, with planned concurrent cisplatin $100 \mathrm{mg} / \mathrm{m}^{2}$ administered intravenously on days 1 and 22 . On both studies, selected patients with base of tongue cancer received a brachytherapy ${ }^{15}$ boost, and surgery was pursued for suspected disease persistence or relapse.

\section{Larynx Preservation Outcome}

Prospective functional data were not available for these patients. As a surrogate measure, successful larynx preservation outcome for this study was defined as local control without any surgery (except biopsy) to the primary site and without a permanent tracheostomy or gastrostomy. Such an endpoint has been successfully used previously. ${ }^{9}$ Overall survival was not included as part of this outcome.

\section{Prognostic Variables}

$\mathrm{T}$ stage, $\mathrm{N}$ stage, and overall TNM stage (by clinical exam alone and with the use of imaging studies to potentially address stage migration concerns ${ }^{16,17}$ ) as well as age, sex, race, primary site, and Karnofsky performance status had all been collected prospectively. After a comprehensive review of the literature, our data set was expanded to include other potential pretreatment prognostic factors, which were collected retrospectively from patient charts. These pretreatment factors included weight loss, ${ }^{18}$ symptoms, ${ }^{19-22}$ comorbidity $^{23}$ (Charlson comorbidity index ${ }^{24}$ ), performance status, ${ }^{25}$ alcohol use,${ }^{18,20}$ cigarette use,${ }^{26-28}$ albumin, ${ }^{18}$ alanine aminotransferase,${ }^{29}$ hemoglobin, ${ }^{29}$ platelets, ${ }^{30}$ mean corpuscular volume, lactic acid dehydrogenase, ${ }^{31}$ serum albumin,${ }^{32}$ and pretreatment tracheostomy $^{21,33}$ and gastrostomy status. All variables were dichotomized before any modeling applying either cutoff points used in the medical literature or based on the frequency distribution in the dataset.

\section{Validation Dataset}

The U.S. Department of Veterans Affairs (VA) Laryngeal Study Group performed a prospective randomized study in patients with stage III or IV larynx cancer. ${ }^{8,33}$ The protocol and informed consent procedures were approved by the institutional review boards at all participating institutions. A total of 332 patients were randomized to receive either standard treatment (surgery and postoperative radiation therapy) or investigational treatment (larynx preservation with sequential standard cisplatin/5-fluorouracil chemotherapy followed by radiation therapy, with primary site surgery reserved for disease persistence or salvage). The full details of treatment plan and outcome have been previously described. ${ }^{8,34}$ Data from the 166 subjects who were randomized to the investigational treatment were used as the validation dataset.

\section{TABLE II.}

Radiation Therapy Regimens.

\begin{tabular}{|c|c|c|c|}
\hline \multicolumn{2}{|c|}{ Radiation Therapy } & \multicolumn{2}{|c|}{ Cisplatin } \\
\hline Target total dose & Fractionation & Dosage & Days \\
\hline \multicolumn{4}{|c|}{ Protocol 1988-1990 $(n=70)$} \\
\hline 6,600-7,000 cGy & $1.8 \mathrm{~Gy} / \mathrm{d}$, wk 1-6 & None & \\
\hline \multicolumn{4}{|c|}{ Protocol 1991-1995 $(n=103)$} \\
\hline 7,000 cGy & $\begin{array}{l}1.8 \mathrm{~Gy} / \mathrm{d}, \text { wk } 1-4 \\
1.8 \mathrm{~Gy} / \mathrm{Am}, \text { wk 5-6 } \\
1.6 \mathrm{~Gy} / \mathrm{PM}, \text { wk 5-6 }\end{array}$ & $100 \mathrm{mg} / \mathrm{m}^{2} \mathrm{IV}$ & 1,22 \\
\hline
\end{tabular}

IV = intravenous. 


\begin{tabular}{lcc}
\hline \multicolumn{3}{c}{ TABLE III. } \\
\hline \multicolumn{3}{c}{ Nemographic Characteristics. } \\
\hline No. of Patients & $\%$ \\
No. analyzed & 173 & - \\
Age, median (range), yr & 170 & - \\
KPS, median (range) & $59(25-84)$ & - \\
Male/female & $80(60-90)$ & - \\
Pretreatment tracheostomy & $123 / 47$ & $72 / 28$ \\
Weight loss $\geq 10 \%$ & 31 & 18 \\
Charlson comorbidity $>3^{*}$ & 28 & 16 \\
Site & 25 & 15 \\
$\quad$ Larynx & & \\
Hypopharynx & 96 & 56 \\
$\quad$ Oropharynx & 47 & 28 \\
Stage & 27 & 16 \\
$\quad$ T2/T3/T4 & & $12 / 43 / 45$ \\
N0/N1/N2/N3 & $21 / 73 / 76$ & $34 / 18 / 45 / 4$ \\
Stage II/III/IV & $57 / 30 / 77 / 6$ & 93 \\
Current/former smokers & $6 / 47 / 117$ & 68 \\
Current/former alcohol users & 53 & \\
\hline \hline
\end{tabular}

*Because of the diagnosis of cancer, all patients had a minimum score of 2.

$\mathrm{KPS}=$ Karnofsky performance status.

\section{Statistics}

Larynx preservation times were calculated from the start of treatment and assigned a value of 0 if local control was never achieved. Univariate analyses of potential prognostic covariates were done using the Kaplan-Meier method. Variables with $P<$ .10 by the log-rank test were included in the multivariate analysis. Multivariate analysis used Cox's proportional-hazards model, ${ }^{35}$ doing the regression in a forward stepwise manner with a threshold significant value of $.10 . P$ values of .10 were used in the early phases of the modeling so that potentially predictive factors with borderline $P$ values were not immediately excluded from the analysis; however, only variables with $P<.05$ after multivariate analysis were included in the final model. Because previous data suggested that patients in the second protocol (1991-1995) had better outcomes than those in the first protocol (1988-1990) ${ }^{36}$ all of the univariate and multivariate analyses were stratified by the protocol in which the patient had participated. The candidate variables for the prognostic tool were derived from the results of the multivariate analysis ( 2 -sided $P$ values $<.05)$. Discrimination was evaluated using the concordance index. ${ }^{37,38} \mathrm{~A}$ concordance index of 1 indicates that a model performs perfectly in predicting success or failure in an individual subject.

Median follow-up for both datasets was computed using the inverse Kaplan-Meier method. ${ }^{39}$ Median as well as actuarial larynx preservation and survival times were reported. For subgroup comparisons of larynx preservation rates, Kaplan-Meier curves were also presented.

\section{RESULTS}

\section{Memorial Sloan-Kettering Cancer Center Development Dataset}

Patient characteristics. The baseline characteristics for those 170 evaluable patients are listed in Table III. The median age was 59 years (range, 25-84), and me- dian Karnofsky performance status was 80 (range, 6090). The majority of primary sites were of the larynx (56\%); $45 \%$ had $\mathrm{N}_{2}$ disease or greater, and $32 \%$ had $\mathrm{T}_{4}$ disease. Fifteen percent of the subjects had significant comorbidity as per the Charlson comorbidity criteria. ${ }^{24}$ A larger proportion were either current/former consumers of tobacco $(93 \%)$ or alcohol $(68 \%)$.

Outcomes. The median follow-up of the patients was 82.5 months (range, 0.4-141.5 months). Fifteen (9\%) were censored before 3 years; 28 (17\%) before 5 years. The median overall survival and time to larynx preservation failure were 54.5 months and 15 months, respectively. The 3 -year overall survival rate was $59 \%$. The 3-year larynx preservation rate was $44 \%$.

Univariate analyses. After analyzing the breakdown of each variable and considering clinically relevant values, each factor was dichotomized as listed in Table IV. Each of the potential prognostic covariates listed in Table IV underwent univariate analysis for larynx preservation outcome. T stage, alcohol use, Karnofsky performance status, lactate dehydrogenase, albumin, platelets, weight loss, pretreatment tracheostomy, and overall stage $(P<.05$ by log-rank test $)$ as well as the symptom of dyspnea $(P<.1)$ were all significant covariates. Cigarette use, either as a dichotomous variable or a continuous variable by pack years (data not shown), was not a significant predictor. The times of last cigarette or alcohol used were also examined both as a continuous variable as well as a dichotomous variable (using 1 month and 1 year as break points), and neither was found to significantly predict successful larynx preservation in our data set.

Multivariate analysis. Two variables that were significant on univariate analysis were excluded from the multivariate analysis. Lactate dehydrogenase was excluded because less than $10 \%$ of the subjects had an abnormally high value pretreatment. Weight loss was excluded owing to concerns about the accuracy of the data using only chart review, particularly the time over which weight loss occurred. All of the other eight variables that were found to predict successful larynx preservation $(P<.10)$ were entered in the Cox's proportional-hazards model. Because of missing data, three subjects (all with larynx cancer as the primary) were excluded from all subsequent analyses. As noted in Table $\mathrm{V}$, four of the variables remained independently significant $(P<.05)$ : T stage $\left(\leq \mathrm{T}_{3}\right.$ vs. $\left.\mathrm{T}_{4}\right)$, serum albumin level $(<4 \mathrm{~g} / \mathrm{dL}$ vs. $\geq 4 \mathrm{~g} / \mathrm{dL})$, Karnofsky performance status ( $<80 \%$ vs. $\geq 80 \%$ ), alcohol/liquor use (described as minor or no alcohol use in the past or $<6$ cans of beer, or the equivalent alcohol, per day during maximum alcohol use vs. described as major alcohol use in the past or $\geq 6$ cans of beer, or the equivalent alcohol, per day during maximum alcohol use). Pretreatment albumin levels had the highest risk ratio (2.55); the risk ratios are similar among the other three variables (1.60-1.77).

TALK score. The risk of unsuccessful larynx preservation was characterized by summing the number of risk factors present at time of diagnosis (Table V). Even though the risk ratio for pretreatment albumin was higher than the other variables, this approach was taken 
TABLE IV.

Univariate Analysis of Pretreatment Factors.

\begin{tabular}{|c|c|c|}
\hline Pretreatment Factors & Dichotomization & $P<.10$ \\
\hline Age, yr & $<60$ vs. $\geq 60$ & * \\
\hline Sex & Male vs. female & * \\
\hline Race & Caucasian vs. non-Caucasian & * \\
\hline Primary site & Larynx vs. hypopharynx or oropharynx & * \\
\hline T stage & $<\mathrm{T} 4$ vs. T4 & $<.001$ \\
\hline $\mathrm{N}$ stage & $<\mathrm{N} 2$ vs. $\geq \mathrm{N} 2$ & * \\
\hline Overall stage & II or III vs. IV & .037 \\
\hline Karnofsky performance status, \% & $<80$ vs. $\geq 80$ & $<.001$ \\
\hline Tracheostomy & Yes vs. no & .004 \\
\hline PEG & Yes vs. no & * \\
\hline \multicolumn{3}{|l|}{ Laboratory serum tests } \\
\hline Lactic acid dehydrogenase & Normal vs. higher than ULN & .027 \\
\hline Albumin, $\mathrm{g} / \mathrm{dL}$ & $<4$ vs. $\geq 4$ & $<.001$ \\
\hline Mean corpuscular volume, $\mathrm{fl}$ & $<100$ vs. $\geq 100$ & * \\
\hline Alanine aminotransferase & Normal vs. higher than ULN & * \\
\hline Hemoglobin, g/dL & $<10$ vs. $\geq 10$ & * \\
\hline Platelets & Normal vs. higher than ULN & .002 \\
\hline Cigarette use & Yes vs. no & * \\
\hline$>40$ pack years & $<40$ vs. $\geq 40$ & * \\
\hline Alcohol use & $\begin{array}{c}\text { Described as minor or no alcohol use in the past or }<6 \text { cans of } \\
\text { beer/d, or the equivalent, during maximum alcohol use vs. described } \\
\text { as major alcohol use in the past or } \geq 6 \text { cans of beer/d, or the } \\
\text { equivalent, during maximum alcohol use }\end{array}$ & .032 \\
\hline Weight loss, \% & $<10$ vs. $\geq 10$ & .002 \\
\hline \multicolumn{3}{|l|}{ Symptoms } \\
\hline Dysphagia & Yes vs. no & * \\
\hline Otalgia & Yes vs. no & * \\
\hline Neck mass & Yes vs. no & * \\
\hline Dyspnea & Yes vs. no & .081 \\
\hline Sore throat & Yes vs. no & * \\
\hline Charlson comorbidity index & $<4$ vs. $\geq 4$ & * \\
\hline
\end{tabular}

${ }^{\star} P>.10$.

$\mathrm{PEG}=$ percutaneous endoscopic gastrostomy; ULN = upper limits of normal.

because there exists overlap of the $95 \%$ confidence intervals of the risk ratios. For example, a patient who had a $\mathrm{T} 4$ tumor (a risk factor), serum albumin level of $3 \mathrm{~g} / \mathrm{dL}$ (a risk factor), a Karnofsky score of 90\% (NOT a risk factor), and never drank alcohol (NOT a risk factor) would have a score of 2 (due to 2 risk factors). Kaplan-Meier curves for each possible TALK score with successful larynx preservation as the outcome are shown in Figure 1. The curves for TALK scores of 1 and 2 were close by visual inspection, so they were combined to represent an intermediate risk for successful larynx preservation. Similarly, the curves for TALK scores of 3 and 4 were close by visual inspection and were therefore combined. Thus, a patient was defined as at good risk if no risk factors were present, intermediate risk if one or two risk factors were present, and poor risk if three or four factors were present.

Furthermore, it was noted that the first letter of each risk factor ( $\mathrm{T}$ stage, albumin, alcohol/liquor use, Karnofsky performance status) made up the acronym TALK. The prognostic model was renamed the TALK score to facilitate the ability to remember the risk factors.
Figure 2A and Table VI summarize larynx preservation rates over time for each prognostic group. The 3-year larynx preservation rates for the good (TALK score $=0$ ), intermediate (TALK score $=1$ or 2 ), and poor (TALK score $=3$ or 4 ) risk groups were $65 \%, 41 \%$, and $6 \%$, respectively. The median time to larynx preservation failure (and the 95\% confidence intervals) for the good-, intermediate-, and poor-risk groups were not reached (117 months: not reached), 14 months (10-37), and 0 months (0-2), respectively. These differences were significant $(\mathrm{P}<.0001$ by log-rank test). All but one patient's cancer in the poor-risk group had larynx preservation failure by 3 years. In the studied population, the majority of the patients $(57 \%)$ fell into the intermediate-risk group; 18 patients $(11 \%)$ were in the poor-risk group.

In the developmental dataset, the concordance index of the TALK score was 0.65 .

TALK score for larynx cancer only. Because only laryngeal cancer patients were part of the validation set, the TALK score model was also applied only to the larynx cancer patients in the Memorial Hospital dataset. 
TABLE V.

Hazard-Cox Forward Stepwise Regression, TALK Score Model.

\begin{tabular}{|c|c|c|c|c|c|}
\hline Variables & Condition & $\operatorname{Pr}>\chi^{2}$ & Hazard Ratio & 95\% Confidence Interval & TALK Score \\
\hline T stage $^{\star}$ & $\mathrm{T}_{4}$ & .008 & 1.76 & $1.16-2.68$ & 1 \\
\hline Albumin & $<4 \mathrm{~g} / \mathrm{dL}$ & .001 & 2.55 & $1.44-4.51$ & 1 \\
\hline Alcohol use (or liquor use) & $\begin{array}{c}\text { Major alcohol use } \\
\text { or }>6 \text { cans of beer/d }\end{array}$ & .023 & 1.60 & $1.07-2.41$ & 1 \\
\hline Karnofsky performance status & $<80 \%$ & .025 & 1.77 & $1.08-2.90$ & 1 \\
\hline
\end{tabular}

*Staging with the use of radiologic evaluation

TALK = T stage, albumin, alcohol/liquor use, Karnofsky performance status.

Overall, there were 93 evaluable subjects with larynx cancer. The 3-year larynx preservation rates (Table VI) for the good- (35 subjects), intermediate- (50 subjects), and poor-risk (8 subjects) groups were $68 \%, 41 \%$, and $13 \%$, respectively. The median time to larynx preservation failure (and the 95\% confidence intervals) for the good-, intermediate-, and poor-risk groups were not reached (lower interval not reached-upper interval not reached), 14 months (9-73), and 0 months (0-8), respectively. These differences were significant $(\mathrm{P}<.0001$ by log-rank test). The larynx preservation Kaplan-Meier curves for the patients with larynx cancer only are illustrated in Figure 2B.

The concordance index of the TALK score in the larynx cancer subgroup was also 0.65 .

\section{Department of Veterans Affairs Laryngeal Cancer Study - Validation Dataset}

Patient characteristics. There were a total of 166 evaluable patients in this population. All subjects in the VA Laryngeal Cancer Study had larynx cancer as the primary site. Of the subjects in this dataset, $26 \%$ had T4 disease; $28 \%$ had N2 or N3 disease; and $44 \%$ had stage IV disease.

Overall outcomes. The median follow-up was 52 months. The actuarial 3-year overall survival rate was $52 \%$. The 3 -year larynx preservation rate was $44 \%$. The median time to larynx preservation failure was 21 months. Compared to the Memorial Hospital data, there

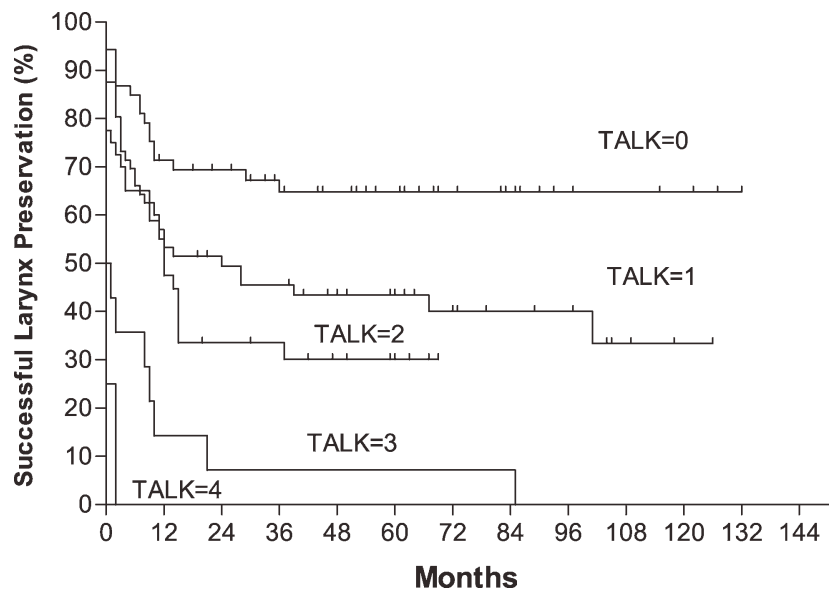

Fig. 1. Kaplan-Meier curves describing larynx preservation rates for each TALK score value. TALK = T stage, albumin, alcohol/liquor use, Karnofsky performance status. were more patients censored early: 25 (15\%) before 3 years and $49(30 \%)$ before 5 years.

TALK score for the VA dataset. When TALK was applied to the VA Laryngeal Cancer Study dataset, 27 subjects $(16 \%)$ were in the good-risk group, $109(66 \%)$ were in the intermediate-risk group, and 30 (18\%) were in the poor-risk group (Table VI). The 3-year larynx preservation rates were $54 \%, 47 \%$, and $25 \%$ for the good-, intermediate-, and poor-risk groups, respectively. The median time to larynx preservation failure (and the 95\% confidence intervals) for the good-, intermediate-,
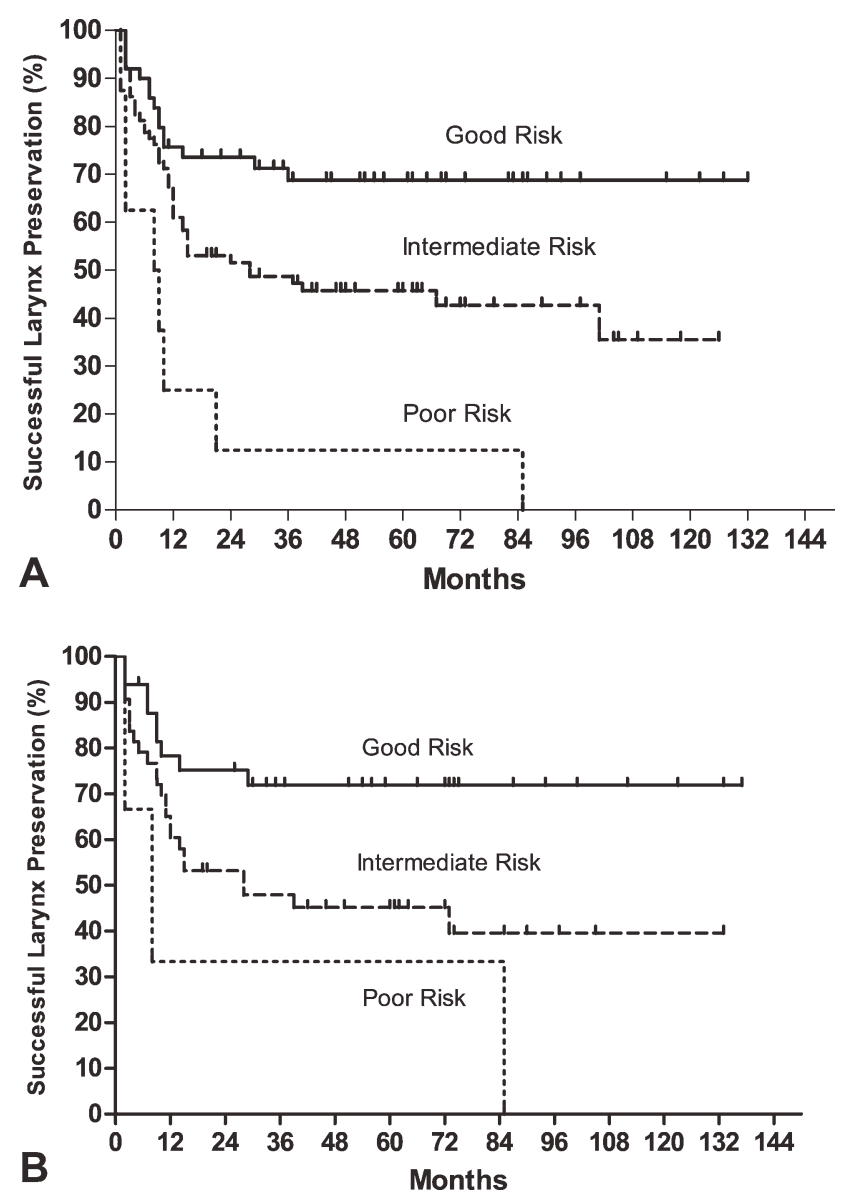

Fig. 2. (A) TALK score model; Memorial Sloan-Kettering Cancer Center data set-all patients. (B) TALK score model; Memorial Sloan-Kettering Cancer Center data set-larynx cancer patients only. TALK $=$ T stage, albumin, alcohol/liquor use, Karnofsky performance status. 
TABLE VI.

TALK Score Groups.

\begin{tabular}{|c|c|c|c|c|c|}
\hline Dataset & TALK Score & Risk Grouping & No.* & Median LP Rate, mo & 3-Year LP Rate, \% \\
\hline \multicolumn{6}{|c|}{ MSKCC (all) } \\
\hline & 0 & Good & 53 & Not reached & 65 \\
\hline & $1-2$ & Intermediate & 96 & 14 & 41 \\
\hline & $3-4$ & Poor & 18 & 0 & 6 \\
\hline & Total & & 167 & 15 & 44 \\
\hline \multicolumn{6}{|c|}{ MSKCC (larynx only) } \\
\hline & 0 & Good & 35 & Not reached & 68 \\
\hline & $1-2$ & Intermediate & 50 & 14 & 41 \\
\hline & $3-4$ & Poor & 8 & 0 & 13 \\
\hline & Total & & 93 & 28 & 49 \\
\hline \multicolumn{6}{|l|}{ VA } \\
\hline & 0 & Good & 27 & 47 & 54 \\
\hline & $1-2$ & Intermediate & 109 & 24 & 47 \\
\hline & $3-4$ & Poor & 30 & 7 & 25 \\
\hline & Total & & 166 & 21 & 44 \\
\hline
\end{tabular}

TALK = T stage, albumin, alcohol/liquor use, Karnofsky performance status; LP = larynx preservation; MSKCC = Memorial Sloan-Kettering Cancer Center; VA = Department of Veterans Affairs Laryngeal Study Group.

and poor-risk groups were 47 months (11; upper interval not reached), 24 months (10; upper interval not reached), and 7 months (2-13), respectively. These differences were significant $(P<.01$ by log-rank test). It should be noted in the actuarial larynx preservation curves provided in Figure 3 that the demarcation at 3 years in the larynx preservation between the good (TALK score $=0$ ) and intermediate (TALK score $=1$ or 2 ) groups did not persist beyond 48 months. Furthermore, seven of the 30 patients in the poor-risk group were censored before the 3-year mark (only 3 patients were censored after 3 years).

In the validation dataset, the concordance index of the TALK score was 0.57 .

\section{DISCUSSION}

The goal of this project was to develop and validate a simple tool that supplies health-care providers and

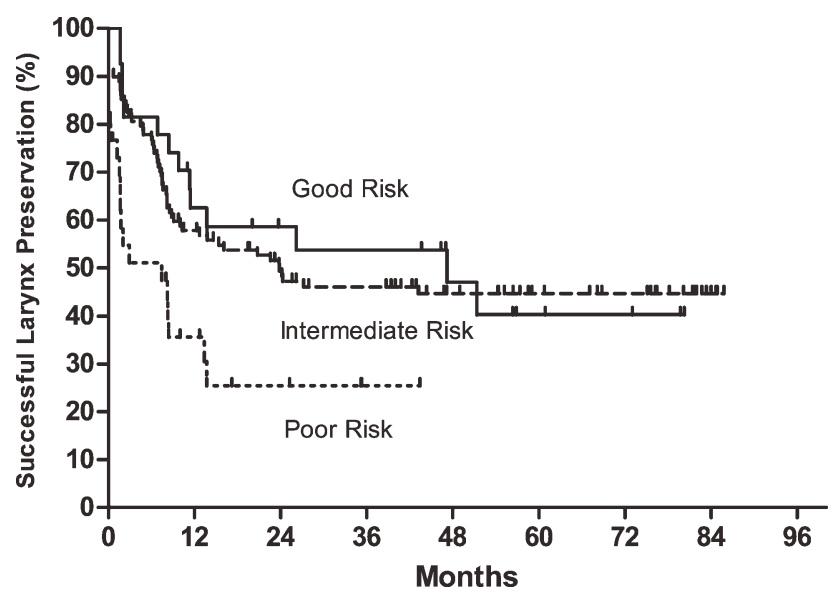

Fig. 3. TALK score model; the Department of Veterans Affairs larynx preservation study. TALK $=\mathrm{T}$ stage, albumin, alcohol/liquor use, Karnofsky performance status. patients with added prognostic data to assist decision making in the larynx preservation setting. The result of our analysis is the TALK score, which demarcated a significant prognostic gradient in larynx preservation outcome in both our (development) and the VA Laryngeal Cancer Study (validation) datasets. The inclusion of the validation step distinguishes our report from other prognostic analyses intended to assist prediction of larynx preservation outcome available in the literature. Numerous clinical factors were analyzed as potential candidates for the prognostic model. Many of these factors, such as comorbidity, symptoms, and alcohol/smoking history, are not routinely collected during the analyses of the management options in patients with cancer. The TALK score consists of $\mathrm{T}$ stage, serum albumin level, alcohol/liquor use, and Karnofsky performance status.

Not surprisingly, the development dataset demonstrated a large gradient between groups. In the validation dataset from the VA Laryngeal Cancer Study, the TALK score's ability to select a poor-risk group, representing nearly $20 \%$ of patients in the study, is also well demonstrated. However, a durable separation of larynx preservation rates between the good- and intermediate-risk groups was not delineated. Potential explanations to consider include a smaller proportion of VA Laryngeal Cancer Study patients in the good-risk group compared to our population (16\% vs. $37 \%$ ) and the impact of approximately $30 \%$ of the VA Laryngeal Cancer Study subjects being censored before 5 years of follow-up compared to $17 \%$ in the Memorial Hospital dataset. Future work will need to identify ways to further delineate between these groups. Arguably, however, the true value of the TALK score is the ability to predict unsuccessful, not successful, larynx preservation. In general, costs and morbidity are higher in subjects who undergo and fail larynx preservation treatment, as they are exposed to additional, potentially toxic, treatments 
that would have been avoided with upfront surgery and adjuvant radiation therapy. Because studies have shown patients to strongly prefer avoiding surgery if possible ${ }^{10,40}$ particularly if survival is not compromised, we anticipate that the majority of patients would choose a larynx preservation option if there exists a reasonable chance of success. Therefore, the clinical impact on decision-making between the good- and intermediate-risk groups is likely much smaller than between the intermediate- and poor-risk groups. The TALK score's ability to identify a poor-risk group is its strength.

The TALK score is simple to use. All variables were dichotomized to facilitate its application. Also, all of the variables are routinely collected by physicians before the initiation of treatment and, for the most part, are independent of high technology. The sole exception to this is the $\mathrm{T}$ stage, which was measured with the aid of a computerized axial tomography scan or magnetic resonance imaging. T stage, based on clinical exam but not imaging, was significant in predicting local control with a functional larynx after multivariate analysis (data not shown) but with a lower relative risk ratio. Nevertheless, most physicians do get a pretreatment imaging study of the primary site. The TALK score provides an objective and valid method to predict the likelihood of successful larynx preservation and an alternative to more subjective, likely less reproducible approaches.

There exist several potentially important applications of the TALK score. First, the TALK score can be used to compare larynx preservation rates from separate nonrandomized larynx preservation trials by allowing investigators to compare the baseline prognostic risks of patients entered in different studies. Second, it will give the patient and clinician additional prognostic data to make an informed decision. However, the health-care provider should not use this model to make a clinical decision independent of the patient. Evidence suggests that health-care providers often gauge patient preferences or quality of life inaccurately. ${ }^{2,41-43}$ Third, if a patient falls into the poor-risk category but truly wishes to pursue a larynx preservation option, an investigational, instead of a standard, larynx preservation approach may be a better treatment choice.

There are certain limitations to this model. The TALK score was created and validated on protocol data sets. Patients participating in clinical trials are required to pass stricter inclusion criteria (see Methods section) than those receiving noninvestigational therapy. Clinical factors that may have importance in a less-selected population may not have been reflected in this clinical trial setting. An example is comorbidity. Although many patients with high comorbidity are excluded from clinical trials, this will not be the case for the head and neck cancer population at large, especially considering the influence of cigarette smoking ${ }^{44}$ and alcohol use ${ }^{45}$ as risk factors for these diseases.

A limitation to the study is the lack of data pertaining to human papillomavirus (HPV) status. It was not until after these studies were completed that HPV was found to be associated with a subset of squamous cell carcinomas of the head and neck. ${ }^{46-48}$ More recent work has shown that HPV-related squamous cell cancers of the head and neck have a favorable prognosis compared to HPV-negative tumors. ${ }^{49,50}$ However, the risk of HPV in non-oropharynx tumors is very low. ${ }^{49,51}$ Considering only $16 \%$ of our development dataset consisted of oropharynx tumors, and there were no oropharynx tumors in our validation dataset, it is unlikely that HPV status is a confounder in our model, although the model is most likely relevant only for HPV-negative tumors.

In addition to HPV, other studies have identified biological markers ${ }^{27,52-56}$ as well as other clinical characteristics $8,33,56$ that may help predict larynx preservation outcome. Examples include the presence of p53 mutations, vascular endothelial growth factor levels, and epidermal growth factor receptor expression. Although the addition of these factors to the TALK score will be considered in future models, the TALK score model in its current form represents a simple and easy to apply model that supplies important information quickly without the aid of difficult, timeconsuming, and/or expensive investigations.

The concordance index of 0.57 for the TALK score when applied to the validation dataset indicated that the model performed better than pure chance in this population but was well below an index of 1 associated with a perfect model. A concordance index in this range is nonetheless consistent with clinical utility. For example, the Gail model, ${ }^{57}$ which is widely used to predict breast cancer risk, has a reported concordance index of $0.58 .^{58}$

\section{CONCLUSION}

The TALK score represents a beginning, a backbone for further prognostic model development in this clinical setting, for which tools to guide management are solely needed. It is likely most relevant to HPV-negative squamous cell cancers of the head and neck. We anticipate further refinement in the scale. For example, other studies have identified biologic markers ${ }^{27,52-56}$ as well as other clinical characteristics ${ }^{8,33,56}$ that may help predict larynx preservation outcome. The addition of selected prognostic factors with the intent of creating a better predictive model while preserving ease of use is being considered for future investigations.

\section{Acknowledgment}

The authors acknowledge the assistance of Michael Kattan, PhD, for his constructive review of the manuscript. Many individuals have been prominently involved in our larynx preservation program over the years, and the authors would particularly like to acknowledge the contributions of Elliot Strong, MD, Ronald Spiro, MD, and Louis Harrison, MD, in this regard.

\section{BIBLIOGRAPHY}

1. Dropkin MJ, Malgady RG, Scott DW, Oberst MT, Strong EW. Scaling of disfigurement and dysfunction in postoperative head and neck patients. Head Neck Surg 1983;6:559-570.

2. Mohide EA, Archibald SD, Tew M, Young JE, Haines T. Postlaryngectomy quality-of-live dimensions identified by patients and health care professionals. Am J Surg 1992;164:619-622.

3. DeSanto LW, Olsen KD, Perry WC, Rohe DE, Keith RL. Quality of life after surgical treatment of cancer of the larynx. Ann Otol Rhinol Laryngol 1995;104:763-769. 
4. Lehmann W, Krebs H. Interdisciplinary rehabilitation of the laryngectomee. Recent Results Cancer Res 1991;121:442-449.

5. Hassmann E, Skotnicka B. Feasibility of supracricoid laryngectomy based on pathological examination. Eur Arch Otorhinolaryngol 1998;255:68-73.

6. Weinstein GS, El-Sawy MM, Ruiz C, et al. Laryngeal preservation with supracricoid partial laryngectomy results in improved quality of life when compared with total laryngectomy. Laryngoscope 2001;111:191-199.

7. Gilbert RW, Birt D, Shulman H, et al. Correlation of tumor volume with local control in laryngeal carcinoma treated by radiotherapy. Ann Otol Rhinol Laryngol 1987;96:514-518.

8. The Department of Veterans Affairs Laryngeal Cancer Study Group. Induction chemotherapy plus radiation compared with surgery plus radiation in patients with advanced laryngeal cancer. $N$ Engl $J$ Med 1991;324:1685-1690.

9. Lefebvre JL, Chevalier D, Luboinski B, Kirkpatrick A, Collette L, Sahmoud T. Larynx preservation in pyriform sinus cancer: preliminary results of a European Organization for Reasearch and Treatment of Cancer phase III trial. J Natl Cancer Inst 1996;88:890-899.

10. Terrell JE, Fisher SG, Wolf GT. Long-term quality of life after treatment of laryngeal cancer. Arch Otolaryngol Head Neck Surg 1998;124:964-971.

11. Pfister DG, Bajorin D, Motzer R, et al. Cisplatin, fluorouracil, and leucovorin. Increased toxicity without improved response in squamous cell head and neck cancer. Arch Otolaryngol Head Neck Surg 1994;120:89-95.

12. Maluf F, Sherman EJ, Kraus DH, et al. Long-term follow-up in patients treated with a larynx preservation approach using sequential chemotherapy and radiation therapy: the Memorial Hospital experience. Acto Oncologica Brasileira 2000;20:78-82.

13. Osman I, Sherman E Singh B, et al. Alteration of p53 pathway in squamous cell carcinoma of the head and neck: impact on treatment outcome in patients treated with larynx preservation intent. J Clin Oncol 2002; 20:2980-2987.

14. Hayes DM, Cvitkovic E, Golbey RB, Scheiner E, Helson L, Krakoff IH High dose cis-platinum diammine dichloride: amelioration of renal toxicity by mannitol diuresis. Cancer 1977;39:1372-1381.

15. Vikram B, Strong E, Shah J, et al. A non-looping afterloading technique for base of tongue implants: results in the first 20 patients. Int $J$ Radiat Oncol Biol Phys 1985;11:1853-1855.

16. Feinstein AR, Sosin DM, Wells CK. The Will Rogers phenomenon: stage migration and new diagnostic technology techniques as a source for misleading statistics for survival in cancer. N Engl J Med 1985;312:1604-1608.

17. Barbera L, Groome PA, Mackillop WJ, et al. The role of computed tomography in the Tclassification of laryngeal carcinoma. Cancer 2001;91:394-407.

18. Mick R, Vokes EE, Weichselbaum RR, Panje WR. Prognostic factors in advanced head and neck cancer patients undergoing multimodality therapy. Otolaryngol Head Neck Surg 1991;105:62-73.

19. Piccirillo JF, Sasaki CT, Wells CK, Feinstein AR. New clinical severity staging system for cancer of the larynx. Ann Otol Rhinol Laryngol 1994 103:83-92.

20. Pugliano FA, Piccirillo JF, Zequeira MR, Fredrickson JM, Perez CA, Simpson JR. Symptoms as an index of biologic behavior in head and neck cancer. Otolaryngol Head Neck Surg 1999;120:380-386.

21. Lassaletta L, Garcia-Pallares M, Morera E, Bernaldez R, Gavilan J. T3 glottic cancer: oncologic results and prognostic factors. Otolaryngol Head Neck Surg 2001;124:556-560.

22. Franchin G, Minatel E, Gobitti C, et al. Radiation treatment of glottic squamous cell carcinoma, stage I and II: analysis of factors affecting prognosis. Int J Radiat Oncol Biol Phys 1998;40:541-548.

23. Singh B, Bhaya M, Zimbler M, et al. Impact of comorbidity on outcome of young patients with head and neck squamous cell carcinoma. Head Neck 1998;20:1-7.

24. Charlson ME, Pompei P, Ales KL, MacKenzie CR. A new method of classifying prognostic comorbidity in longitudinal studies: development and validation. J Chronic Dis 1987;40:373-383.

25. Cognetti F, Pinnaro P, Ruggeri EM, et al. Prognostic factors for chemotherapy response and survival using combination chemotherapy as initial treatment of advanced head and neck squamous cell cancer. J Clin Oncol 1989;7:829-837.

26. Brennan JA, Boyle JO, Koch WM, et al. Association between cigarette smoking and mutation of the p53 gene in squamous-cell carcinoma of the head and neck. N Engl J Med 1995;332:712-717.

27. Temam S, Flahault A, Perie S, et al. p53 gene status as a predictor of tumor response to induction chemotherapy of patients with locoregionally advanced squamous cell carcinomas of the head and neck. $J$ Clin Oncol $2000 \cdot 18: 385-394$

28. De Boer MF, Van den Borne B, Pruyn JF, et al. Psychosocial and physical correlates of survival and recurrence in patients with head and neck carcinoma: results of a 6-year longitudinal study. Cancer 1998;83:2567-2579.

29. Himberlin C, Merol JC, Nasca S, et al. Prognostic significance of routine clinical and laboratory data in advanced head and neck cancers. Anticancer Res 1996;16:1005-1010.

30. Huang GW, Nong HT, Yu QS, Kinjoh K, Nakamura M, Kosugi T. Platelet aggregation in head and neck tumors in China. Laryngoscope 1997;107: $1142-1145$.
31. Cheng SH, Jian JJ, Tsai SY, et al. Prognostic features and treatment outcome in locoregionally advanced nasopharyngeal carcinoma following concurrent chemotherapy and radiotherapy. Int $J$ Radiat Oncol Biol Phys 1998;41:755-762.

32. Medow MA, Weed HG, Schuller DE. Simple predictors of survival in head and neck squamous cell carcinoma. Arch Otolaryngol Head Neck Surg $2002 ; 128: 1282-1286$

33. Wolf G, Hong W, Fisher S, et al. Larynx preservation with induction chemotherapy and radiation in advanced laryngeal cancer: final results of the VA laryngeal cancer study group cooperative trial. Proc Amer Soc Clin Oncol 1993;12:277.

34. Spaulding MB, Fischer SG, Wolf GT. Tumor response, toxicity, and survival after neoadjuvant organ-preserving chemotherapy for advanced laryngeal carcinoma. The Department of Veterans Affairs Cooperative Laryngeal Cancer Study Group. J Clin Oncol 1994;12:1592-1599.

35. Cox DR. Regression models and life-tables. J R Stat Soc Series B Stat Methodol 1972;34:187-220.

36. Zahalsky AJ, Sherman EJ, Kraus D, et al A multivariate assessment of concomitant boost $(\mathrm{CB})$ radiation therapy (RT) and cisplatin versus conventional RT as part of a larynx preservation (LP) strategy. Proc Am Soc Clin Oncol 2001;20:(Abstract 898).

37. Harrell FE Jr, Califf RM, Pryor DB, Lee KL, Rosati RA. Evaluating the yield of medical tests. JAMA 1982;247:2543-2546.

38. Begg CB, Cramer LD, Venkatraman ES, Rosai J. Comparing tumour staging and grading systems: a case study and a review of the issues, using thymoma as a model. Stat Med 2000;19:1997-2014.

39. Schemper M, Smith TL. A note on quantifying follow-up in studies of failure time. Control Clin Trials 1996;17:343-346.

40. McNeil BJ, Weichselbaum R, Pauker SG. Speech and survival: tradeoffs between quality and quantity of life in laryngeal cancer. $N$ Engl J Med 1981;305:982-987

41. DiNardo LJ, Kaylie DM, Isaacson J. Current treatment practices for early laryngeal carcinoma. Otolaryngol Head Neck Surg 1999;120:30-37.

42. Bjordal K, Freng A, Thorvik J, Kaasa S. Patient self-reported and clinician-rated quality of life in head and neck cancer patients: a cross-sectional study. Eur J Cancer B Oral Oncol 1995;31B:235-241.

43. Slevin ML, Stubbs L, Plant HJ, et al. Attitudes to chemotherapy: comparing views of patients with cancer with those of doctors, nurses, and general public. BMJ 1990;300:1458-1460.

44. Spitz M. Epidemiology and risk factors for head and neck cancer. Semin Oncol 1994;21:281-288.

45. Saracci R. The interactions of tobacco smoking and other agents in cancer etiology. Epidemiol Rev 1987;9:175-193.

46. Gillison ML, Koch WM, Shah KV. Human papillomavirus in head and neck squamous cell carcinoma: are some head and neck cancers a sexually transmitted disease? Curr Opin Oncol 1999;11:191-199.

47. Gillison ML, Koch WM, Capone RB, et al. Evidence for a causal association between human papillomavirus and a subset of head and neck cancers. J Natl Cancer Inst 2000;92:709-720.

48. Gillison ML, Lowy DR. A causal role for human papillomavirus in head and neck cancer. Lancet 2004;363:1488-1489.

49. Fakhry C, Westra WH, Li S, et al. Improved survival of patients with human papillomavirus-positive head and neck squamous cell carcinoma in a prospective clinical trial. J Natl Cancer Inst 2008;100:261-269.

50. Ang KK, Harris J, Wheeler R, et al. Human papillomavirus and survival of patients with oropharyngeal cancer. N Engl J Med 2010;363:24-35.

51. Gillison ML, D'Souza G, Westra W, et al. Distinct risk factor profiles for human papillomavirus type 16-positive and human papillomavirus type 16-negative head and neck cancers. J Natl Cancer Inst 2008;100: 407-420.

52. Bradford CR, Zhu S, Wolf GT, et al. Overexpression of p53 predicts organ preservation using induction chemotherapy and radiation in patients with advanced laryngeal cancer. Department of Veterans Affairs Laryngeal Cancer Study Group. Otolaryngol Head Neck Surg 1995;113:408-412.

53. Gregg CM, Beals TE, McClatchy KM, Fisher SG, Wolf GT. DNA content and tumor response to induction chemotherapy in patients with advanced laryngeal squamous cell carcinoma. Otolaryngol Head Neck Surg 1993;108:731-737.

54. Johnston PG, Mick R, Recant W, et al. Thymidylate synthase expression and response to neoadjuvant chemotherapy in patients with advanced head and neck cancer. J Natl Cancer Inst 1997;89:308-313.

55. Teknos TN, Cox C, Barrios MA, et al. Tumor angiogenesis as a predictive marker for organ preservation in patients with advanced laryngeal carcinoma Laryngoscope 2002;112:844-851.

56. Staton J, Robbins KT, Newman L, Samant S, Sebelik M, Vieira F. Factors predictive of poor functional outcome after chemoradiation for advanced laryngeal cancer. Otolaryngol Head Neck Surg 2002;127:43-47.

57. Gail MH, Brinton LA, Byar DP, et al. Projecting individualized probabilities of developing breast cancer for white females who are being examined annually. J Natl Cancer Inst 1989;81:1879-1886.

58. Rockhill B, Spiegelman D, Byrne C, Hunter DJ, Colditz GA. Validation of the Gail et al. model of breast cancer risk prediction and implications or chemoprevention. $J$ Natl Cancer Inst 2001:93:358-366. 